\title{
TRATAMENTO CIRÚRGICO PARA HARPEJAMENTO IDIOPÁTICO EM EQUINO: relato de caso
}

\section{Surgical treatment of idiopathic stringhalt in a horse: case report}

\author{
Camila Giunco $^{1} \mathbb{D}_{\text {; }}$ Rogério Navarro de Abreu $^{2}$ \\ *Autor Correspondente: Camila Giunco. Rua Dom João Nery, 141, \\ Nova Paulínia, Paulínia, SP, Brasil. CEP: 13140-305. \\ E-mail: camila.giuncovet@gmail.com
}

\begin{abstract}
Como citar: GIUNCO, C.; ABREU, R. N. Tratamento cirúrgico para harpejamento idiopático em equino: relato de caso. Revista de Educação Continuada em Medicina Veterinária e Zootecnia do CRMV-SP, São Paulo, v. 19, n. 1, e38130, 2021. DOI: https://doi.org/10.36440/recmvz.v19i1.38130.

Cite as: GIUNCO, C.; ABREU, R. N. Surgical treatment of idiopathic stringhalt in a horse: case report. Journal of Continuing Education in Veterinary Medicine and Animal Science of CRMV-SP, São Paulo, v. 19, n. 1, e38130, 2021. DOl: https://doi.org/10.36440/recmvz.v19i1.38130.
\end{abstract}

\section{Resumo}

O harpejamento é uma anormalidade caracterizada pela hiperflexão involuntária de um ou dos dois membros pélvicos, manifestando-se como claudicação de intensidade e frequência diversificada, variando de grau leve a severo. Essa enfermidade pode ocorrer por traumas no membro pélvico, exercício repetitivo, além de intoxicação por ingestão de plantas tóxicas, porém não apresenta etiologia bem definida. Pode ser classificado como harpejamento australiano ou harpejamento clássico/idiopático, apresentando sinais clínicos semelhantes, porém a primeira forma diferencia-se da segunda pela gravidade, ocorrência em surtos, sazonalidade e recuperação espontânea dos animais acometidos. 0 tratamento pode ser medicamentoso, com terapias complementares, ou por intervenção cirúrgica. 0 prognóstico é de reservado a favorável de acordo com o quadro clínico do animal. Este trabalho descreve um caso de harpejamento idiopático com enfoque na apresentação clínica e forma de tratamento.

Palavras-chave: Arpejo. Hiperflexão. Claudicação.

\section{Abstract}

Stringhalt is an abnormality characterized by involuntary hyperflexion of one or both hindlimb with lameness of different intensity and frequency, varying from mild to severe. The cause of this disease can be trauma, repetitive exercise and poisoning by ingesting toxic plants, although it has not a defined etiology. It is classified as Australian stringhalt or classic/idiopathic stringhalt, and in both of

1 Aprimoranda em Clínica Médica e Cirúrgica em Grandes Animais, Fundação de Ensino Octávio Bastos, Centro Universitário, Hospital Veterinário Dr. Vicente Borelli, São João da Boa Vista, SP, Brasil

2 Docente do curso de Medicina Veterinária, Fundação de Ensino Octávio Bastos, Centro Universitário, São João da Boa Vista, SP, Brasil 
them the clinical sings are similar. The first one differs from the second by: severity, outbreak occurrence and spontaneous recovery of the affected horse. Treatment can be conservative, with complementary therapies, or by surgical intervention. The prognosis is guarded to favorable according to the patient clinical condition. This study describes a case of idiopathic stringhalt focusing on clinical presentation and treatment.

Keywords: Stringhalt. Hyperflexion. Lameness.

\section{Introdução}

O harpejamento é uma hiperflexão involuntária do tarso, com apresentação uni ou bilateral, que ocorre quando o animal está em movimento (SULLINS apud BAXTER, 2011). Conhecido também como hipertonia reflexa equina (DUQUE et al., 2014), pode ser um sinal clínico de desequilíbrio funcional de músculos flexores e extensores que transpõem o tarso. (MARTINEZ, 2007). No decorrer da fase cranial da passada, o membro é flexionado em direção ao abdômen, podendo haver variações na gravidade dos sinais a cada passada (PELOSO apud AUER; STICK, 2012).

A causa do harpejamento geralmente é indefinida, podendo estar relacionada a um fator neurológico, seja pela lesão de nervos periféricos ou pela toxicidade de plantas (BROCKMAN, 2016), além de inflamação por lesão no tarso e intoxicação por alcaloides (latirismo) (MARTINEZ, 2007). A enfermidade apresenta-se de duas formas principais, a primeira é geralmente unilateral, com casos isolados, também conhecida como harpejamento clássico (LIMA; FARIAS; BAPTISTA FILHO, 2014) ou idiopático, podendo ocorrer de forma espontânea, por exercícios repetitivos ou traumas localizados no tendão, gerando processo inflamatório e aderência das estruturas envolvidas, entre outras lesões nos tecidos moles da região do tarso (BAXTER, 2011; MARTINEZ, 2007; SULLINS, 2011) Santos et al. (2019) referem o desenvolvimento de harpejamento, durante o período de recuperação, de um equino com laceração parcial do tendão digital profundo.

Peloso apud Auer; Stick (2012) ressaltam que a verdadeira etiologia do harpejamento clássico é desconhecida, porém sugerem a neuropatia subjacente pois, por histologia, pode evidenciar a neuropatia ou miopatia degenerativa em biópsia de músculos e nervos periféricos de cavalos com harpejamento idiopático.

A segunda forma do harpejamento é a bilateral, conhecida como harpejamento australiano ou adquirido, que ocorre de forma sazonal, em surtos ou casos isolados (MARTINEZ, 2007). Esta forma tem sido relatada na Austrália, Nova Zelândia, América do Norte, Brasil, Itália, Chile e Japão (PELOSO apud AUER; STICK, 2012), podendo ser resultado da exposição a neurotoxinas e subsequente neuropatia periférica, ou a neurotoxinas derivadas de plantas (EL-HAGE et al., 2017) do tipo Taraxacum officinale, Malva parviflora, Hypochaeris radicata, Rumex acetosella, Elymus repens, que predominam nas pastagens em épocas de seca (BAXTER, 2011; BROCKMAN, 2016; DEL PIERO; ROBERTSON, 2015; PELOSO, 2012; SULLINS, 2011). Acredita-se que possa ocorrer uma neuropatia periférica (axonopatia) e atrofia muscular neurogênica do extensor digital longo e lateral, músculo gastrocnêmio e cricoaritenóideo dorsal (BAXTER, 2011; PELOSO, 2012). Sullins apud Baxter (2011) salienta que as plantas anteriormente descritas estão fortemente associadas à doença, porém, ainda não foram definitivamente consideradas como causas diretas.

Tanto no harpejamento idiopático quanto no australiano, os sinais clínicos são similares, embora o primeiro tenha maior impacto em um único membro pélvico (DEL PIERO; ROBERTSON, 2015). Os sinais são variáveis, alguns animais apresentam suave aumento de flexão do tarso, ao passo que outros mostram um movimento mais acentuado do boleto em direção ao abdômen, nos casos mais graves pode haver contato da face dorsal do boleto com a porção ventral do abdômen (SULLINS apud BAXTER, 2011). As alterações podem se mostrar a cada passo ou de maneira espasmódica, e a flexão se torna mais evidente quando o animal recua ou realiza um movimento circular (MARTINEZ, 2007). A mudança 
climática pode interferir na intensidade do movimento, pois o tempo frio agrava os sinais, e o clima quente ameniza a hiperflexão (SULLINS apud BAXTER, 2011). A fisiopatologia da hiperflexão ainda não é bem descrita e a hipótese mais aceita é de que no membro acometido os músculos extensores debilitados têm a sua ação sobreposta pelos flexores, minimamente afetados (BAXTER, 2011; LIMA; FARIAS; BAPTISTA FILHO, 2014).

O diagnóstico do harpejamento é baseado no exame clínico, observando-se os sinais de hiperflexão de um ou dos dois membros pélvicos, variando entre graus mais leves aos mais severos. Todas as raças podem ser afetadas (PELOSO apud AUER; STICK, 2012). O diagnóstico diferencial deve considerar a miopatia fibrótica, fixação dorsal de patela e espasmos musculares (SULLINS apud BAXTER, 2011), além de osteoartrite (esparavão), lesões na região proximal do metatarso e enfermidades que promovem dores no casco podendo levar à hiperflexão e à alteração funcional do tarso (MARTINEZ, 2007).

A recuperação espontânea do harpejamento australiano foi relatada após o isolamento da pastagem e repouso absoluto do animal por semanas, podendo chegar a até um ano. Por outro lado, o harpejamento clássico não apresenta melhora espontânea (BAXTER, 2011). Martinez (2007) refere que apesar da melhora espontânea, tratamentos com uso de miorrelaxantes, anti-inflamatórios não esteroides e infiltração com corticoides foram realizados, porém não apresentaram resultados favoráveis, levando ao tratamento cirúrgico (miotenectomia do extensor digital lateral), por apresentar resultado muitas vezes imediato ou em curto prazo. Já Brockman (2016) obteve resultados satisfatórios com o emprego da acupuntura.

A técnica de miotenectomia tem a finalidade de remover 2 a $10 \mathrm{~cm}$ da região distal do músculo extensor digital lateral e de todo o seu tendão (PELOSO, 2012). o procedimento pode ser feito com o animal sob anestesia geral em decúbito lateral, ou sedado e em pé, com anestesia local onde são realizadas as incisões (KRAMER apud WILSON, 2006). A primeira incisão é feita na face dorsolateral, sobre o tendão, na região proximal à junção com o tendão extensor digital longo, seguida da dissecação do local e exposição da estrutura com pinça Kelly curva ou Ochsner. A segunda incisão, também na porção dorsolateral do membro é longitudinal, com 6 a $10 \mathrm{~cm}$ de extensão, acima do maléolo lateral da tíbia e se estende pelos planos de pele, tecido subcutâneo e fáscia sobre o músculo extensor digital lateral. Após a identificação e dissecação, o músculo é exposto com o emprego de um instrumento cirúrgico curvo. Antes de realizar a tenectomia, deve-se averiguar se o músculo e o tendão, isolados nas duas incisões, são correspondentes. 0 tendão é seccionado na incisão distal e, com uma tesoura Mayo ou pinça Ochsner, o músculo é tracionado para remover o tendão de sua bainha e exteriorizá-lo totalmente. Forçar a tração pode ser necessário, pois podem existir aderências em torno do tendão. Posteriormente, incisa-se o músculo na porção mais proximal da incisão, assegurando que pelo menos $2 \mathrm{~cm}$ de músculo seja removido. Para finalizar, na incisão proximal, é feita a fasciorrafia, com padrão de sutura simples contínuo com fio no 0 sintético absorvível. A pele é suturada, nas duas incisões, com fio não absorvível sintético com padrão simples contínuo. Por fim, é feito o curativo nas feridas cirúrgicas e confinamento do animal em baia por 10 a 14 dias, até a remoção das suturas. (DUQUE et al., 2014; KRAMER, 2006; PELOSO, 2012; SULLINS, 2011). Após a realização do procedimento cirúrgico se faz necessária a aplicação de bandagem nos membros operados, com troca a cada dois a quatro dias. Até que seja retirada a sutura, o animal deve ficar estabulado por pelo menos duas semanas e com restrição de exercício (KRAMER, 2006). No pós-operatório, Duque et al. (2014) indicam a realização da profilaxia medicamentosa com o emprego de penicilina benzatina (15.000 UI/ $\mathrm{kg}$, IM, dose única) e Fenilbutazona (4,4 mg/kg, IV, SID, por oito dias).

Nos casos de harpejamento idiopático, o prognóstico situa-se entre reservado a favorável, e usualmente ocorre a melhora após a realização do procedimento cirúrgico, porém, o nível de melhora nos sinais não é previsível. Para o harpejamento australiano o prognóstico é semelhante e muitos cavalos apresentam recuperação após remoção da pastagem, porém outros não apresentam uma melhora espontânea (BAXTER, 2011). 


\section{Relato de caso}

Um animal da espécie equina, da raça manga-larga, macho não castrado, de $380 \mathrm{~kg}$, utilizado para passeio, com 16 anos de idade, foi atendido em hospital veterinário privado, com histórico de 60 dias de claudicação dos membros pélvicos, mostrando exacerbada flexão do tarso. 0 proprietário informou que o animal vivia estabulado, era vacinado contra raiva, não havia sido vermifugado e era alimentado com ração ( $2 \mathrm{~kg} / \mathrm{dia}$ ) e feno do tipo Coast-Cross (à vontade) e água limpa e abundante.

Os parâmetros fisiológicos e os valores hematológicos e bioquímicos do animal não apresentaram alterações. 0 exame específico de sistema locomotor foi realizado com foco nos membros pélvicos, não havendo alteração no exame estático e no pinçamento dos cascos. Na inspeção dinâmica do animal ao passo foi observada hiperflexão dos tarsos, mais severa no membro pélvico direito, chegando ao diagnóstico clínico de harpejamento. Optou-se pelo tratamento cirúrgico dos dois membros pélvicos, com o emprego da técnica de miotenectomia do extensor digital lateral.

Para o procedimento cirúrgico foi realizada a tricotomia na porção lateral do terço médio da tíbia até distal de metatarso, o animal foi anestesiado com: xilazina $(1 \mathrm{mg} / \mathrm{kg})$ na MPA, cetamina $(2,2 \mathrm{mg} / \mathrm{kg})$ e diazepam $(0,2 \mathrm{mg} / \mathrm{kg})$ para indução anestésica, manutenção com isofluorano (vaporizador calibrado $1,4 \%)$ e infusão contínua de cetamina $(0,5 \mathrm{mg} / \mathrm{kg} / \mathrm{h})$. 0 animal foi colocado em decúbito dorsal e a antissepsia cirúrgica foi realizada com clorexidina degermante e alcoolico, a área foi isolada com campo cirúrgico estéril. A primeira incisão longitudinal, de aproximadamente $2 \mathrm{~cm}$, foi executada diretamente sobre o tendão extensor digital lateral, próximo a sua junção com o tendão extensor digital longo. Em seguida, fez-se a dissecação local para identificação do tendão, e com uma pinça Kelly curva, o tendão foi isolado e exposto. A segunda incisão, longitudinal e na mesma direção das fibras musculares, de aproximadamente $7 \mathrm{~cm}$, foi efetuada diretamente sobre o músculo extensor digital lateral. Após a identificação, a fáscia foi incisada e o músculo foi dissecado, exposto e isolado com o emprego de uma pinça Rochester Pean curva (Figura 1). Após o isolamento do músculo e tendão, o músculo foi tracionado para a certificação de que as estruturas das duas incisões eram correspondentes. Seguiu-se com a tenotomia distal, tração e exteriorização do tendão extensor digital lateral (Figura 2) pela incisão proximal. Após esta etapa, foi realizada a secção do músculo na porção proximal da incisão, garantindo a remoção de pelo menos $2 \mathrm{~cm}$ do músculo (Figura 3). Para finalizar, na incisão proximal, foi realizada a fasciorrafia com fio sintético absorvível 2-0 e plano de sutura simples contínuo, seguido de sutura de pele com fio não absorvível sintético 0 e plano de sutura Sultan. Na incisão distal, foi executada apenas a sutura de pele com fio não absorvível sintético e plano de sutura Sultan. A seguir procedeu-se o curativo local e bandagem. Logo após a recuperação anestésica, o animal apresentou melhora de $50 \%$ no andamento. No terceiro dia após o procedimento cirúrgico, o animal apresentou melhora clínica da claudicação em $100 \%$. 
Figura 1 - Exposição do músculo extensor digital lateral (A) e tendão extensor digital lateral (B) para realização da técnica de miotenectomia em equino

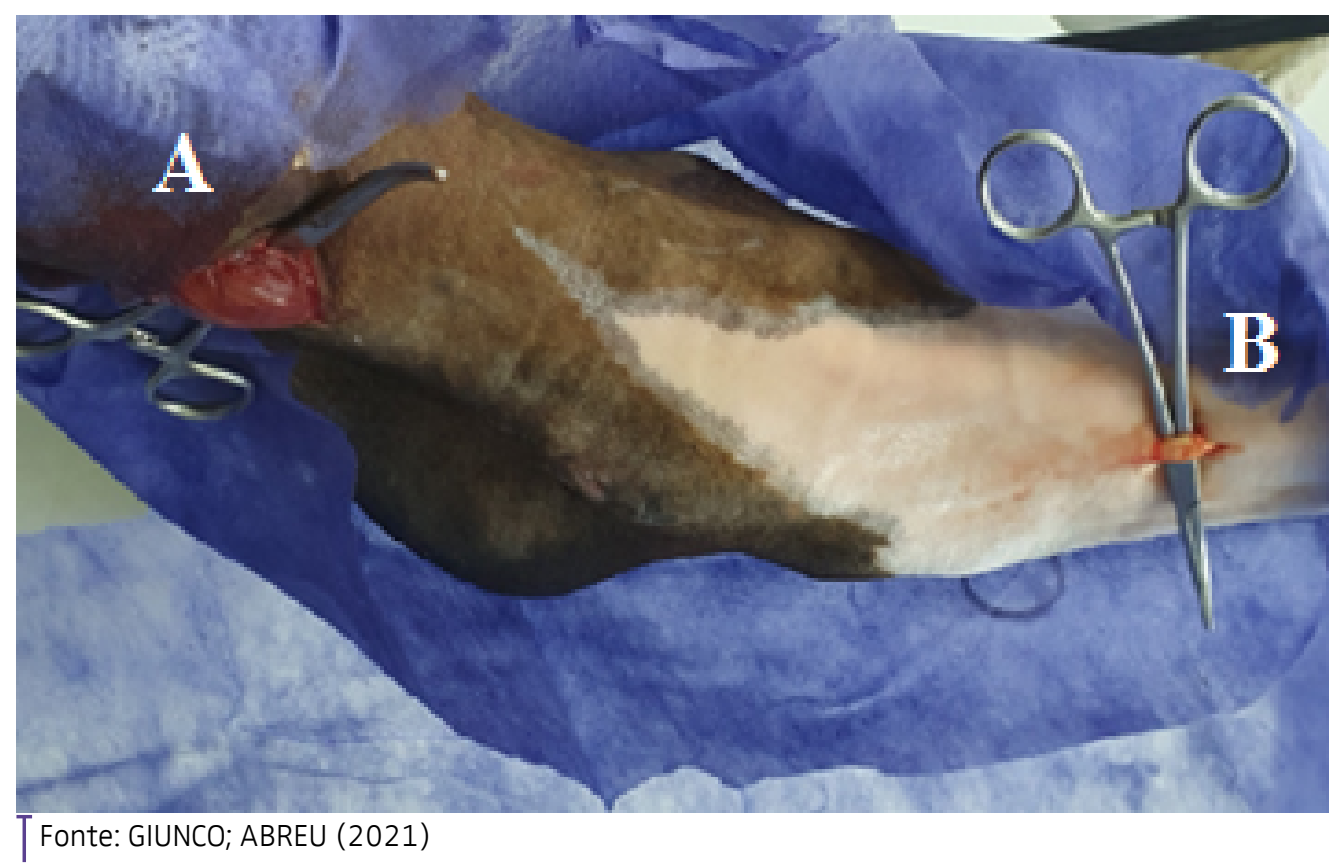

Figura 2 - Tenectomia (A) e exteriorização do tendão extensor digital lateral (B) em equino

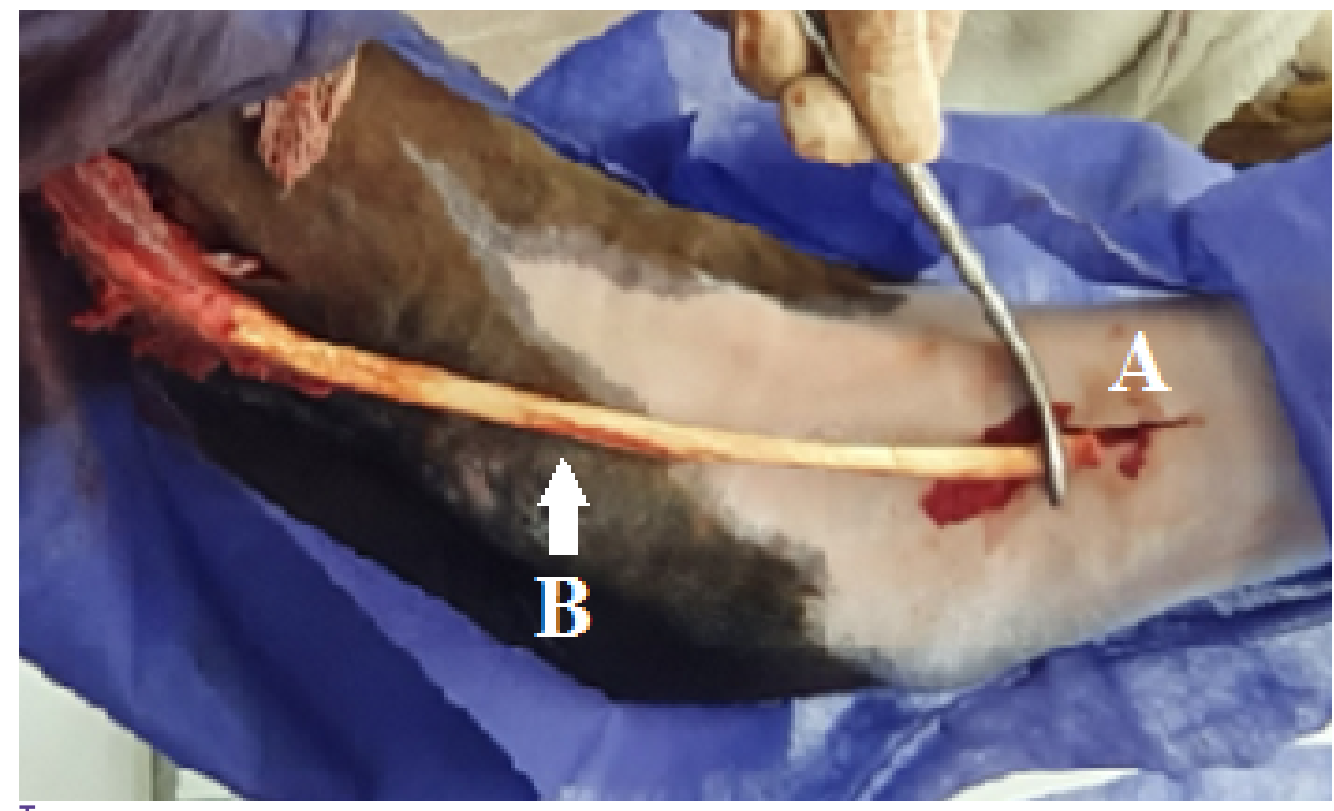

T Fonte: GIUNCO; ABREU (2021) 
Figura 3 - Porção removida de tendão (A) e musculatura (B) no procedimento de miotenectomia do extensor digital lateral em equino

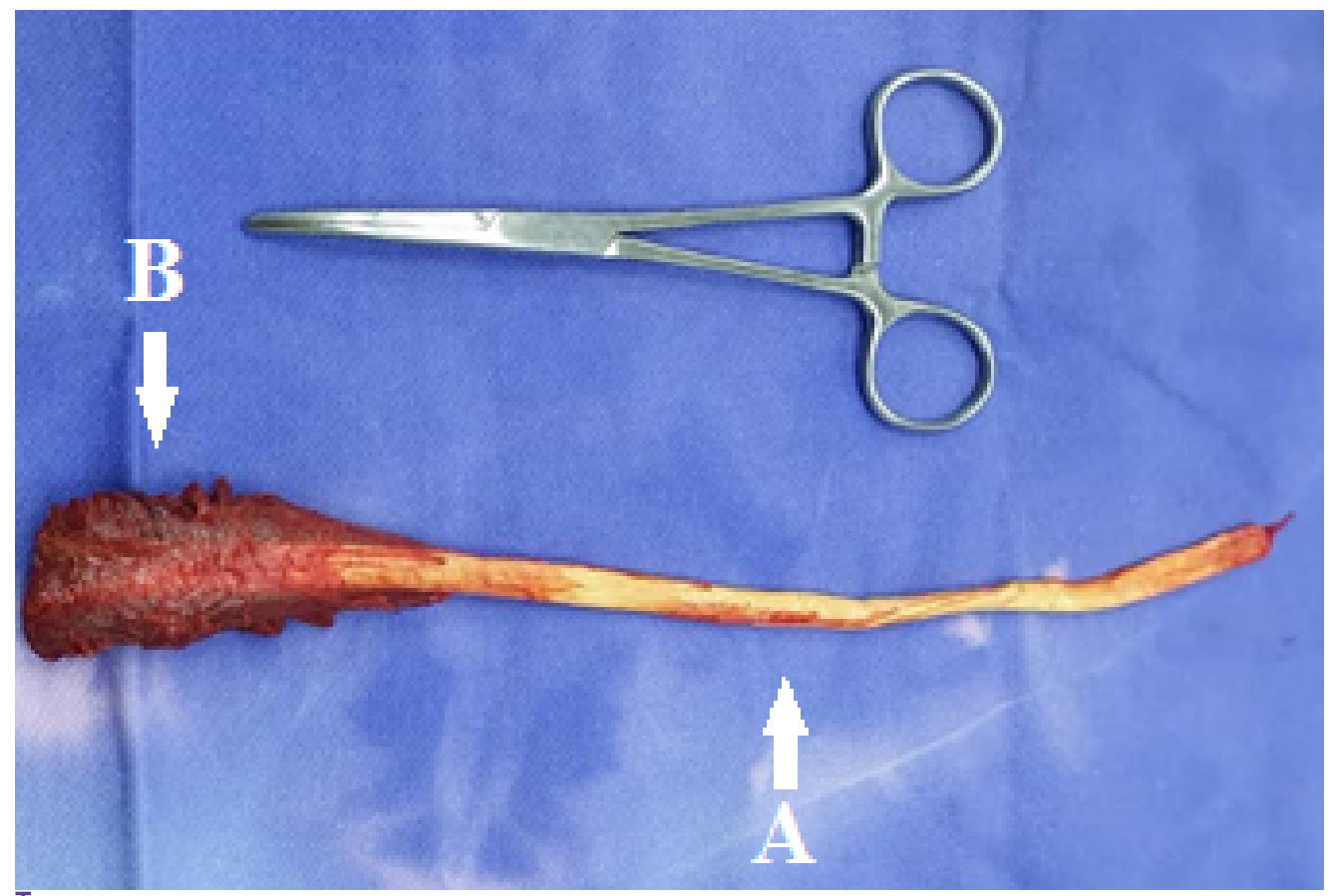

Fonte: GIUNCO; ABREU (2021)

No período pós-operatório o tratamento instituído foi enrofloxacina (10 mg/kg; VO; SID; 7 dias), primeira dose oferecida antes do procedimento cirúrgico, flunixina meglumina, (1,1mg/kg; IV; SID; 5 dias), antes da sedação do animal, omeprazol (1g; VO; SID; 7 dias), instituiu-se profilaxia antitetânica com soro antitetânico (5000 UI, IM ), ducha local (duas vezes ao dia), limpeza da ferida cirúrgica com clorexidina degermante (duas vezes ao dia), curativo local com pomada antimicrobiana (Furanil) e spray prata (duas vezes ao dia), além de caminhadas diárias.

Após oito dias de internação, o animal recebeu alta hospitalar, mantendo o tratamento com antibiótico por mais três dias. Tal conduta foi adotada com o antibiótico, profilaxia por longo período, devido às condições sanitárias precárias do ambiente em que o animal vivia. Concluídos 15 dias de pós-operatório, o animal retornou ao hospital para retirada dos pontos, avaliação da ferida cirúrgica e inspeção dinâmica dos membros pélvicos. 0 resultado foi favorável, não houve deiscência na ferida cirúrgica e o animal não apresentou claudicação.

\section{Discussão}

Os sinais clínicos do caso relatado, associados à anamnese, conduziram ao diagnóstico de harpejamento clássico, pois o animal apresentou, de maneira crônica e progressiva, hiperflexão dos tarsos, sendo o membro pélvico direito o mais acometido, não havendo histórico de intoxicação por plantas da família Asteraceae, além de ausência de histórico de traumas na região do tarso ou doenças anteriores. Duque et al. (2014) relataram um caso de harpejamento idiopático semelhante ao do presente trabalho, onde o animal não havia ingerido plantas tóxicas e não apresentava histórico de traumas no membro pélvico, em que o diagnóstico definitivo foi firmado com base na anamnese, observação dos sinais clínicos e exame de eletromiografia dos músculos dos membros pélvicos. Entretanto, Martinez (2007), ressalta que para o diagnóstico de harpejamento clássico ser estabelecido, além do histórico clínico e exame estático e dinâmico das estruturas do tarso, também devem ser considerados os diagnósticos diferenciais de: fixação de patela, osteoartrite, mieloencefalite protozoária equina, miopatia fibrótica 
ou outras lesões dos membros pélvicos que desencadeiam dor e consequente alteração na flexão do tarso. No presente caso, estas afecções foram descartadas.

Devido ao relato de progressiva e exacerbada flexão do tarso direito do animal e dificuldade de locomoção ao passo, optou-se pelo tratamento cirúrgico com miotenectomia do extensor digital lateral. Para Sullins apud Baxter (2011), a recuperação espontânea dos animais acometidos pelo harpejamento clássico não é comum, e a miotenectomia distal do extensor digital lateral deve ser o tratamento de eleição. 0 uso de ultrassom terapêutico, acupuntura, repouso, analgésicos e infiltração articular com corticoides têm sido propostos, porém não têm apresentado resultados satisfatórios (SULLINS, 2011). Duque et al. (2014) notaram que o tratamento medicamentoso não ofereceu bons resultados para um caso grave de harpejamento idiopático, não havendo regressão dos sinais clínicos, com isso, optou-se pela intervenção cirúrgica, a qual resultou em melhora clínica aos 30 dias da realização do procedimento.

No presente caso, a miotenectomia, de ambos os membros pélvicos, foi realizada em centro cirúrgico, com o animal sob anestesia geral e posicionado em decúbito dorsal, contudo Sullins (2011) descreveu o procedimento cirúrgico realizado com o animal em posição quadrupedal ou em decúbito lateral em mesa cirúrgica. No presente trabalho e no de Peloso (2012), a técnica cirúrgica adotada promoveu a remoção de 2 a $10 \mathrm{~cm}$ da região distal do músculo extensor digital lateral e de todo o seu tendão.

A terapia medicamentosa pós-cirúrgica, empregada no caso descrito, utilizou flunixina meglumina (1,1 mg/kg; IV; SID; 5 dias), que apresenta poder anti-inflamatório e analgésico; enrofloxacina (10 mg/kg; VO; SID; 7 dias), utilizada como antibiótico profilaxia de amplo espectro; omeprazol (1g; VO; SID; 7 dias), para proteção gástrica; e profilaxia antitetânica, com o uso de 5000 UI de soro por via intramuscular. Já, para o mesmo procedimento de miotenectomia, Duque et al. (2014) utilizaram apenas fenilbutazona $(4,4 \mathrm{mg} / \mathrm{kg}$; IV; SID; 8 dias), com poder anti-inflamatório e analgésico, e penicilina benzatina (dose única de $15000 \mathrm{UI} / \mathrm{kg}$ ) como antibioticoprofilaxia. Enquanto, Kramer apud Wilson (2006) indicou apenas o uso de fenilbutazona ( $4,4 \mathrm{mg} / \mathrm{kg}$, BID, IV, por $1 \mathrm{dia}$ ), pois considerava desnecessário o uso de antibiótico como terapia ou profilaxia. No presente caso, a antibiótico-profilaxia por período prolongado foi instituída para prevenir o desenvolvimento de infecções, devido às condições higiênico-sanitárias deficitárias do local onde $o$ animal era mantido.

0 resultado do procedimento cirúrgico adotado no presente caso foi favorável, pois o animal apresentou melhora aproximada de $50 \%$ nos sinais clínicos imediatamente após a recuperação anestésica, recuperando-se plenamente no terceiro dia pós-cirúrgico. Resultados semelhantes foram referidos por Lima et al. (2014), onde o animal também apresentou melhora no andamento após o procedimento, não havendo complicações pós-operatórias, voltando a caminhar normalmente em três semanas. Porém, Martinez (2007) relata que a resposta ao tratamento cirúrgico pode ser diversa, existindo casos de recidiva e não melhoria dos sinais clínicos.

Para o caso aqui relatado, a decisão pela miotenectomia se mostrou acertada e o prognóstico foi favorável, com recuperação total do andamento do animal em três dias após o procedimento cirúrgico, no entanto, Duque et al. (2014) relataram casos em que a melhora progressiva na claudicação, com recuperação completa foi observada apenas depois de um ano da realização do procedimento cirúrgico. De fato, Kramer (2006) ressalta que embora os resultados positivos do tratamento cirúrgico do harpejamento sejam recompensadores, eles podem variar e não são previsíveis, sendo que a melhora no quadro clínico, quando presente, pode ocorrer no período pós-cirúrgico imediato, em dias ou no decurso de meses. 


\section{Conclusão}

Para o caso relatado, a opção de tratamento cirúrgico do harpejamento pela miotenectomia do extensor digital lateral foi acertada e eficaz, promovendo a completa resolução no andamento do animal em três dias após o procedimento, sem complicações pós-operatórias e alta hospitalar em oito dias de internação.\&

\section{Referências}

BAXTER, G. M. Common conditions of the hindlimb. In: BAXTER, G. M. Manual of equine lameness. 1st. ed. Oxford: Wiley Blackwell, 2011. p. 357-358.

BROCKMAN, T. A case study utilizing myofascial release, acupressure and trigger point therapy to treat bilateral "Stringhalt" in a 12-year-old Akhal-Teke horse. Journal of Bodywork \&

Movement therapies, United States of America, v. 21, n. 3, p. 589-593, Set. 2016. DOI: https://doi. org/10.1016/j.jbmt.2016.08.019.

DEL PIERO, F.; ROBERTSON, J. L. The basics of equine neuropathology. In: FURR, M.; REED, S. Equine neurology. 2nd. ed. Oxford: Wiley Blackwell, 2015, p. 203.

DUQUE, D. et al. Idiopathic stringhalt in a Colombian Creole horse. Revista Colombiana de Ciencias Pecuarias, Colombia, v. 27, p. 227-233, Jan. 2014.

EL-HAGE, C. M. et al. Pasture-associated stringhalt: contemporary appraisal of an enigmatic syndrome. Equine Veterinary Education, Australia, v. 31, n. 3, p. 154-162, Apr. 2017. DOl: https://doi. org/10.1111/eve.12751.

KRAMER, J. Lateral digital extensor tenectomy. In: WILSON, D. A et al. Manual of equine field surgery. Missouri: Elsevier, 2006. p. 72-75.

LIMA, J. T. B.; FARIAS, A. F. A.; BAPTISTA FILHO, L. C. F. Tratamento cirúrgico de harpejamento clássico em equino: relato de caso. Ciência Veterinária nos Trópicos, Recife, v. 17, n. 3, p. 130, set./dez. 2014. MARTINEZ, J. Considerações sobre a claudicação mecânica caracterizada por hiperflexão do tarso: arpejamento. Revista Brasileira de Medicina Equina, [s.I.], v. 14, p. 4, jan. 2007.

PELOSO, J. G. Biology and management of muscle disorders and diseases. In: AUER, J. A.; STICK, J. A. Equine surgery. 4th. ed. Missouri: Elsevier, 2012. p. 1182-1184.

SANTOS, G. Y. et al. Laceração parcial de tendão flexor digital profundo com desenvolvimento de harpejamento em equinos: relato de caso. Revista UNINGÁ Review, Maringá, v. 34, n. 1, p. 34, jun./ set. 2019.

SULLINS, K. E. Lameness in the extremities. In: BAXTER, G. M. Adams \& Stashak's lameness in horses. 6th. ed. Oxford: Wiley Blackwell, 2011. p. 1178-1180.

TRecebido: 8 de fevereiro de 2021. Aprovado: 28 de junho de 2021. 